ob manche der seltenen Elemente die ihnen bis jetzt zugeschriebenen Atomgewichte in der That besitzen. In einer folgenden Abhandlung sollen zunächst die in dieser Beziehung gemachten Beobachtungen über das Erbium mitgetheilt werden.

Zum Schlufs erfülle ich noch die angenehme Pflicht, den Assistenten am hiesigen Laboratorium, Herren Dr. Paul Kies ew et ter und Karl Hofmann, für die ausgezeichnete Unterstützung zu danken, welche dieselben mir bei Ausführung obiger Untersuchung zu Theil werden liefsen.

\title{
Ueber die Capillaritätsconstanten organischer Stoffe in wässerigen Lösungen;
}

\section{von J. Traube.}

Bisher sind von den auf dem Gebiete der Capillarität thätigen Forschern, wie auch von mir in früheren Arbeiten, nur die Capillaritätsconstanten $\mathbf{a}^{2}$ und $\alpha(=\gamma)$ in Betracht gezogen worden, Constanten, deren Gröfse durch die bekannten Capillaritätsgleichungen $\mathrm{a}^{2} \cos \vartheta=\mathrm{r} \mathrm{h}$ und $\alpha \cos \vartheta$ $=\frac{\mathrm{rhs}}{2}$ gegeben ist.

Diese Constanten bilden ein Mafs für die Cohäsion der gesammten Lösung. Es schien mir hier jedoch wünschenswerth, diejenige Methode, welche sich für andere physikalische Eigenschaften so fruchtbar erwiesen hat, auch auf die Capillarität auszudehnen, d. h. molekulare Lösungen zu untersuchen, und durch Subtraction der Constanten der Lösung von denjenigen des Wassers Constanten zu gewinnen, welche den Einflufs zeigen, den die gelösten Moleküle ihrer Qualität, Zahl und Gröfse nach auf die Capillarität ausüben.

Diese Betrachlungsweise schien mir um so mehr Berechtigung zu haben, als vom rein kinetischen Standpunkte aus die Grôfse der Capillaritätsconstante einer Lösung sich zusammensetzen würde aus den Druckgröfsen, welche durch die 
Stöfse der auf die Flächeneinheit in der Zeiteinheit anprallenden Moleküle von Lösungsmittel + gelöster Substanz gegeben sind.

Durch Aufstellung obiger Constanten (Lösungsmittel Lösung) erhält mau demnach ein Mafs für die von den gelösten Molekülen ausgeübten Capillarkräfte. Sofern mit Aenderung der Concentration keine Dissociations- oder Associationsvorgänge verknüpft waren, $d$. $h$. die gelösten Moleküle von einheitlicher Gröfsenordnung blieben, mufsten, wie vorauszusehen war, jene von mir aufgestellten Capillaritätsconstanten $a^{2}{ }_{w}-a^{2}{ }_{1}=e^{2}$ und $\alpha_{w}-\alpha_{1}=\varepsilon$ der Concentration $c, d . h$. der Anzahl der gelösten Moleküle, proportional wachsen, d. h. die aus Concentration und Capillarität gebildeten Producte c. $\mathrm{e}^{\mathrm{P}}$ und c. $\varepsilon$ mufsten constant sein. Diese Constanz besagt nichts anderes als die Gültigkeit eines Gesetzes für verdünnte Lösungen, welches demjenigen von Boyle, für Basen entspricht. Mit zunehmender Concentration mufsten, in dem Malse als eine Association der Jonen nach Arrhenius, bezw. der Moleküle zu gröfseren Complexen nach älterer Anschauung vor sich ging, auch die Constanten $\mathrm{c}^{2}$ bez. $\mathrm{c} \varepsilon \mathrm{ab}-$ nehmen; aus der Grölse dieser Abnahme, sowie Fesstellung derjenigen Verdünnung, bei welcher die Constanz des Ausdruckes $\mathrm{c} \mathrm{e}^{2}$ bez. $\mathrm{c} \varepsilon$ eintritt, liefsen sich Schlüsse erwarten, die für die Fesstellung der Dissociations- oder Associationsvorgänge in Lösungen nicht ohne Bedeutung waren. Die Constanten $\mathrm{c} \mathrm{e}^{2}$ und $\left.\mathrm{c} \varepsilon^{*}\right)$, welche vollständig den Werthen $\mu$ des molekularen Leitvermögens entsprechen, wurden von mir mit dem Namen der specifischen und wirklichen Molekularcohäsion bezeichnet.

Bei meinen Versuchen bediente ich mich des Capillari-

*) Die Werthe $\theta^{2}$ und $\varepsilon$ enthalten jedoch sehr wahrscheinlich noch eine vom Randwinkel $\vartheta$ abhängige Function, indem wir schwerlich berechtigt sind $\cos \vartheta=1$ zu setzen. Vgl. meine früheren Arbeiten Journ. f. pract. Chem. N. F. 31, 514, 1885 und 84, 292 u. f. 1886. 
meters *), seltener und nur zur Controle des Stalagmometers. Die früher erwähnten Vorsichtsmafsregeln **) wurden auf's sorgfältigste beobachtet. Jede Beobachtung der Steighöhe $\mathbf{h}$ wurde wenigstens dreimal wiederholt, Abweichungen von $0,3 \mathrm{~mm}$ wurden hierbei selten beobachtet. Ich beschränke mich auf Veröffentlichung der Mittelwerthe der Steighöhen. Zu sämmtlichen Beobachtungen diente dieselbe, sehr gleichmäfsige Röhre. Ihr Radius $\mathbf{r}$ wurde bestimmt (mittelst Quecksilber) zu $0,1771 \mathrm{~mm}$ und $0,1769 \mathrm{~mm}$, also $0,177 \mathrm{~mm}$ angenommen. Die specifischen Gewichte $s$ wurden (nach der Formel $\left.s=\frac{m}{w}(Q-\lambda)+\lambda\right)$ mittelst des $S p r$ eng el'schen Pyknometers bestimmt.

Der besseren Uebersichtlichkeit halber finden sich unter $\delta$ die durch die gelösten Moleküle bewirkten specifischen Gewichtserhöhungen bezw. Erniedrigungen, bezogen auf Wasser.

Die Zimmertemperatur war sehr angenähert stets $=15^{\circ} \mathrm{C}$, die Schwankungen bewegten sich innerhalb $0,5^{\circ} \mathrm{C}$. (Maximalfehler auf $\mathrm{h}=0,1 \mathrm{~mm}$.

Für die Reinheit der von Kahlbaum Berlin bezogenen Substanzen bürgte in vielen Fällen die Bestimmung des specifischen Gewichtes, wie auch des electrolytischen Leitvermögens ; die Concentrationen wurden selbstverständlich mit grofser Sorgfalt hergestellt. $c=1,2,4,8 \ldots$ stellt Lösungen dar, welche 1,1/8,1/4,1/8 . . . Gramn - Molekulargewichte im Liter der Lösung enthalten.

Wenn sich auch über die Gröfse des Gesammtfehlers bei meinen Versuchen nicht in allen Fällen etwas sicheres aussagen läfst, so sind die relativen Fehler doch höchst unbedeutend; die absoluten Werthe von $\mathrm{a}^{2}$, auf die es mir hier allerdings weniger ankam, wurden gegenüber meinen früheren Versuchen um 0,1 bis $0,2 \mathrm{~mm} \mathrm{zu}$ klein gefunden.

*) Journ. f. pract. Chem. N. F. 81, 177, 1885.

**) Fresenius, Zeitschr. f. analyt. Chem. $28,26,1888$. 


\begin{tabular}{|c|c|c|c|c|}
\hline$\stackrel{9}{1}$ & 0 & $\begin{array}{l}0,0000 \\
+10000\end{array}$ & 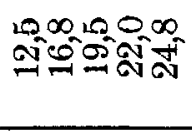 & 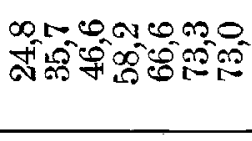 \\
\hline$\prod_{\substack{\infty \\
0 \\
0 \\
0}}^{0}$ & 0 & 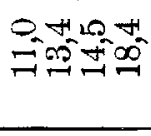 & 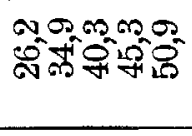 & कू⿻ \\
\hline$\overbrace{\omega}^{\infty}$ & 0 & $\begin{array}{l}x_{0} 0000 \\
09100\end{array}$ & 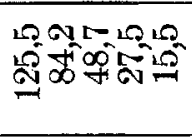 & 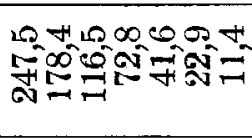 \\
\hline 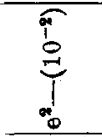 & 0 & $\begin{array}{l}0090 \\
00000\end{array}$ & की & 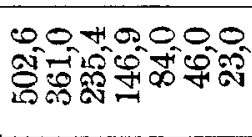 \\
\hline 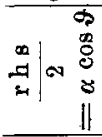 & 葛 & 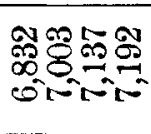 & 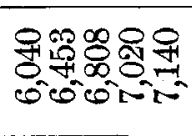 & 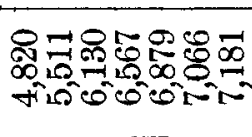 \\
\hline 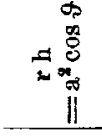 & 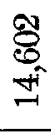 & 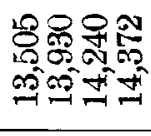 & 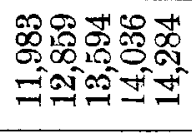 & 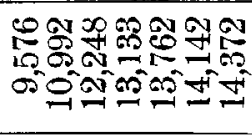 \\
\hline تص & की & $\cos _{\infty}^{\infty}=\overbrace{\infty}^{\infty}$ & $\begin{array}{l}-0005 r \\
000000\end{array}$ & 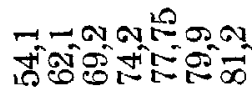 \\
\hline $\begin{array}{l}\stackrel{p}{b} \\
\dot{0} \\
\dot{0}\end{array}$ & 0 & 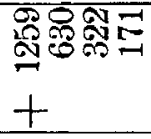 & 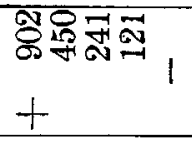 & 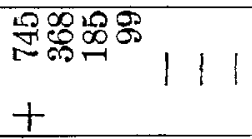 \\
\hline$\stackrel{0}{\infty}$ & कू & 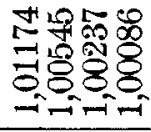 & 요요 & 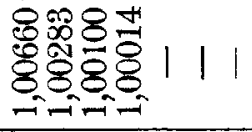 \\
\hline 0 & ! & $\neg \infty+\infty$ & $-0+\infty \notin$ & 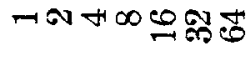 \\
\hline & 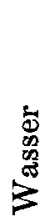 & 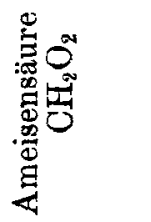 & 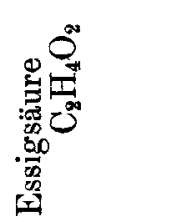 & 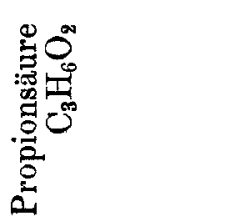 \\
\hline
\end{tabular}


O M O 60 H

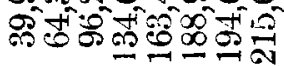

H $-\infty, 000$. पी
ब. सดी

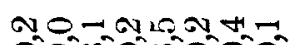
बंक्षिक की का कर

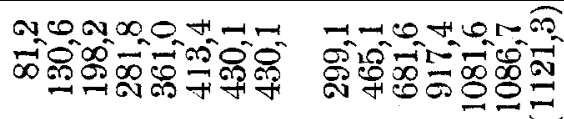

$027-1=202000$

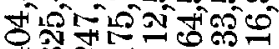

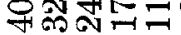

- Mt कीज कीजि जक्षण

ककू

सजभी

$\infty+0,000$

$\infty 0-1-\infty 20,0$

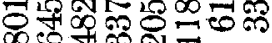

की

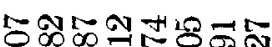

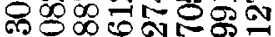
मीं 200000

if

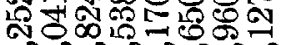

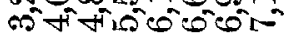

काने L क

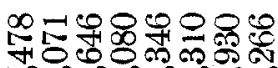
co

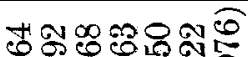
12 5 - 00 \% m सं०ि०0.0.0

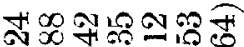
- 2 की nó

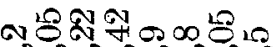
का

$\cos c 0 \cos 2 \mathrm{~N}$ 영 92.200

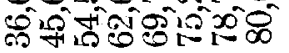

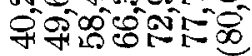

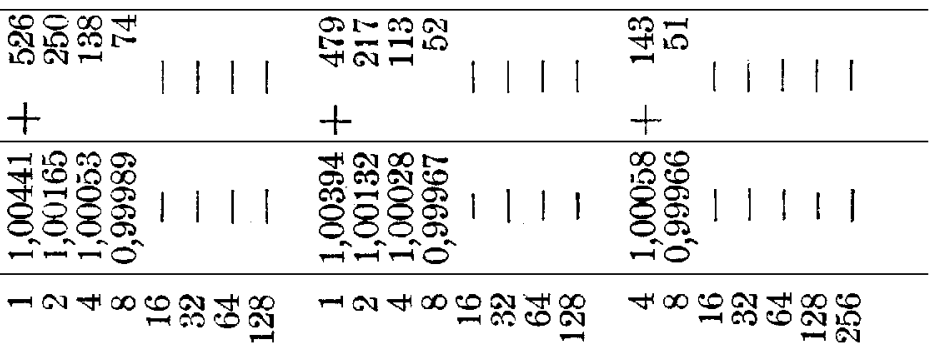
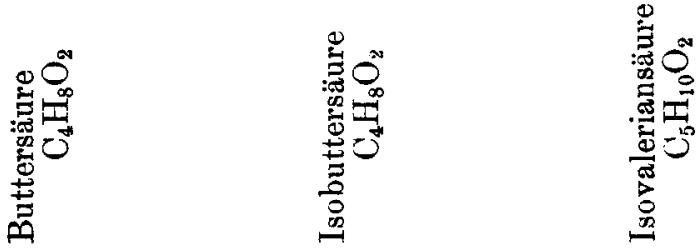


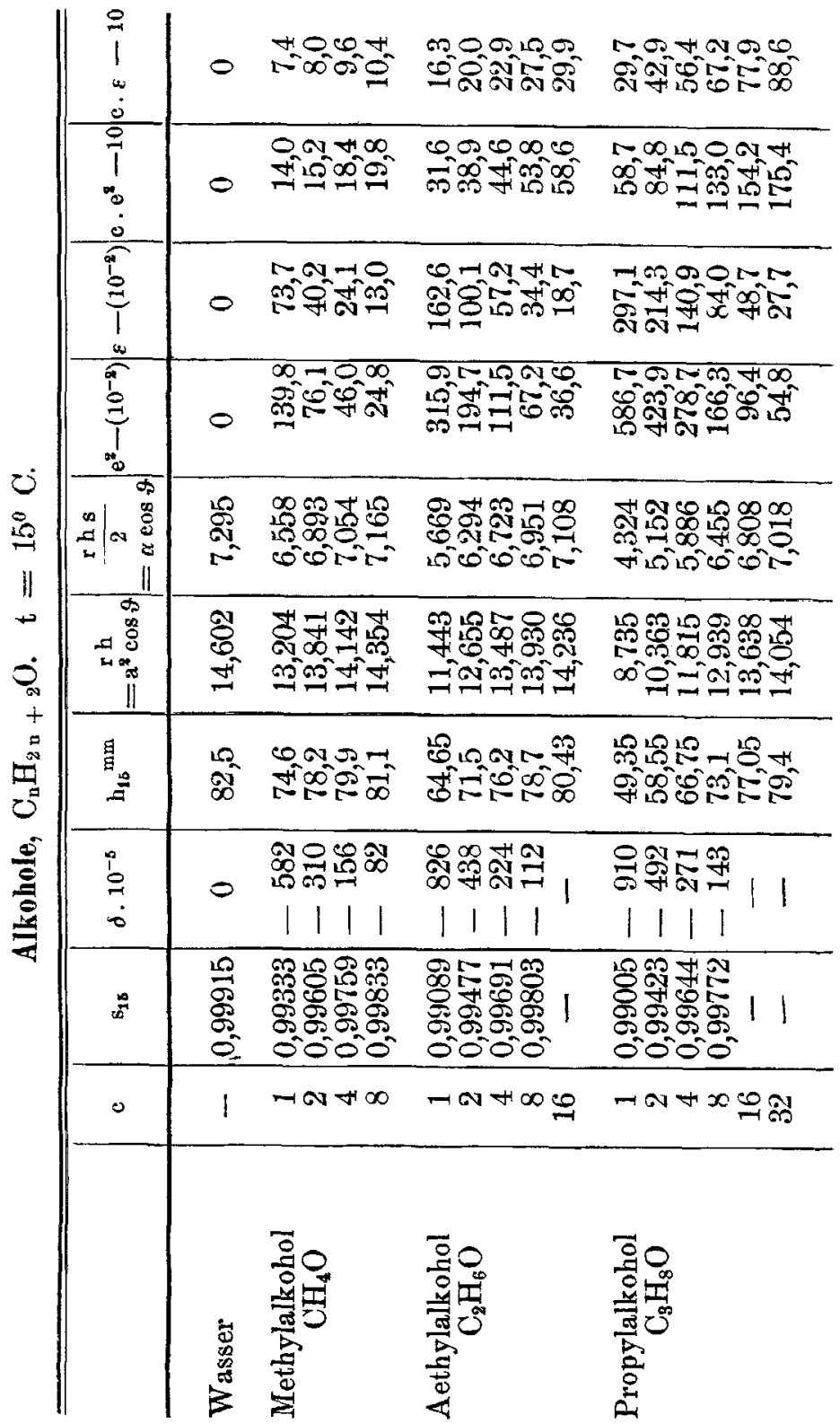




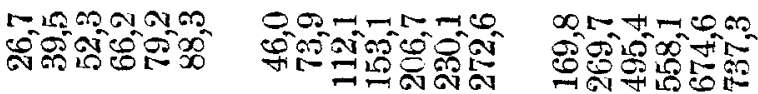

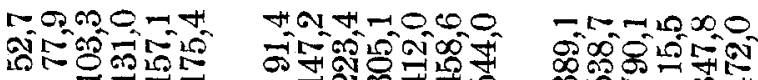

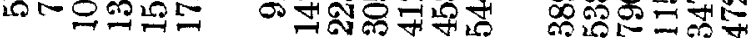

SOSt

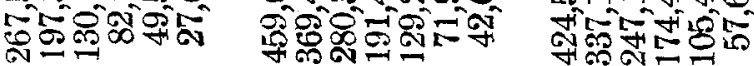

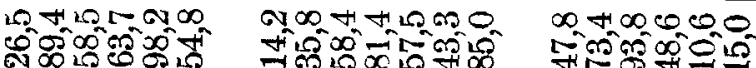

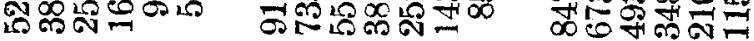

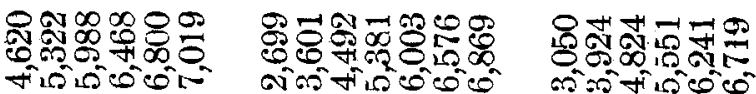

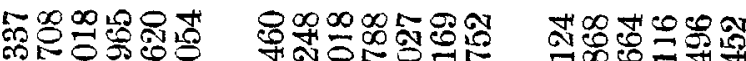

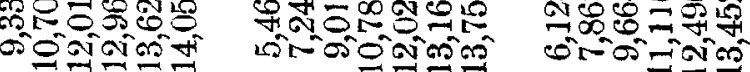

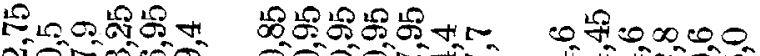

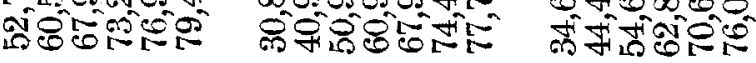

\begin{tabular}{|c|c|c|}
\hline 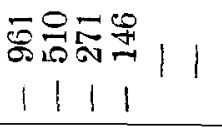 & 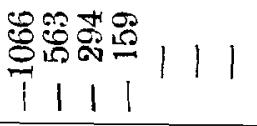 & $\begin{array}{l}\text { क्षे } \\
11\end{array}$ \\
\hline 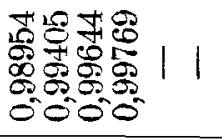 & 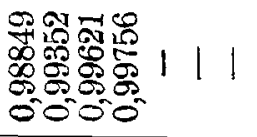 & 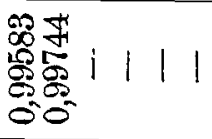 \\
\hline 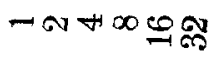 & -Nस & 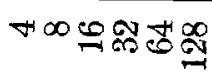 \\
\hline
\end{tabular}
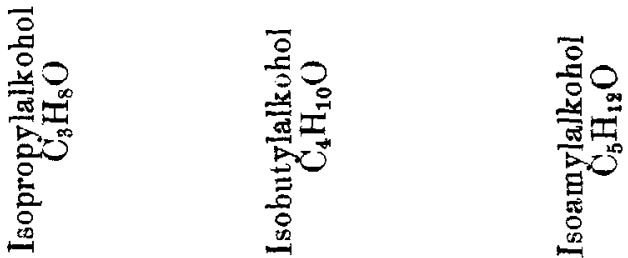

Armalen der Chemie $465 . \mathrm{Bd}$ 


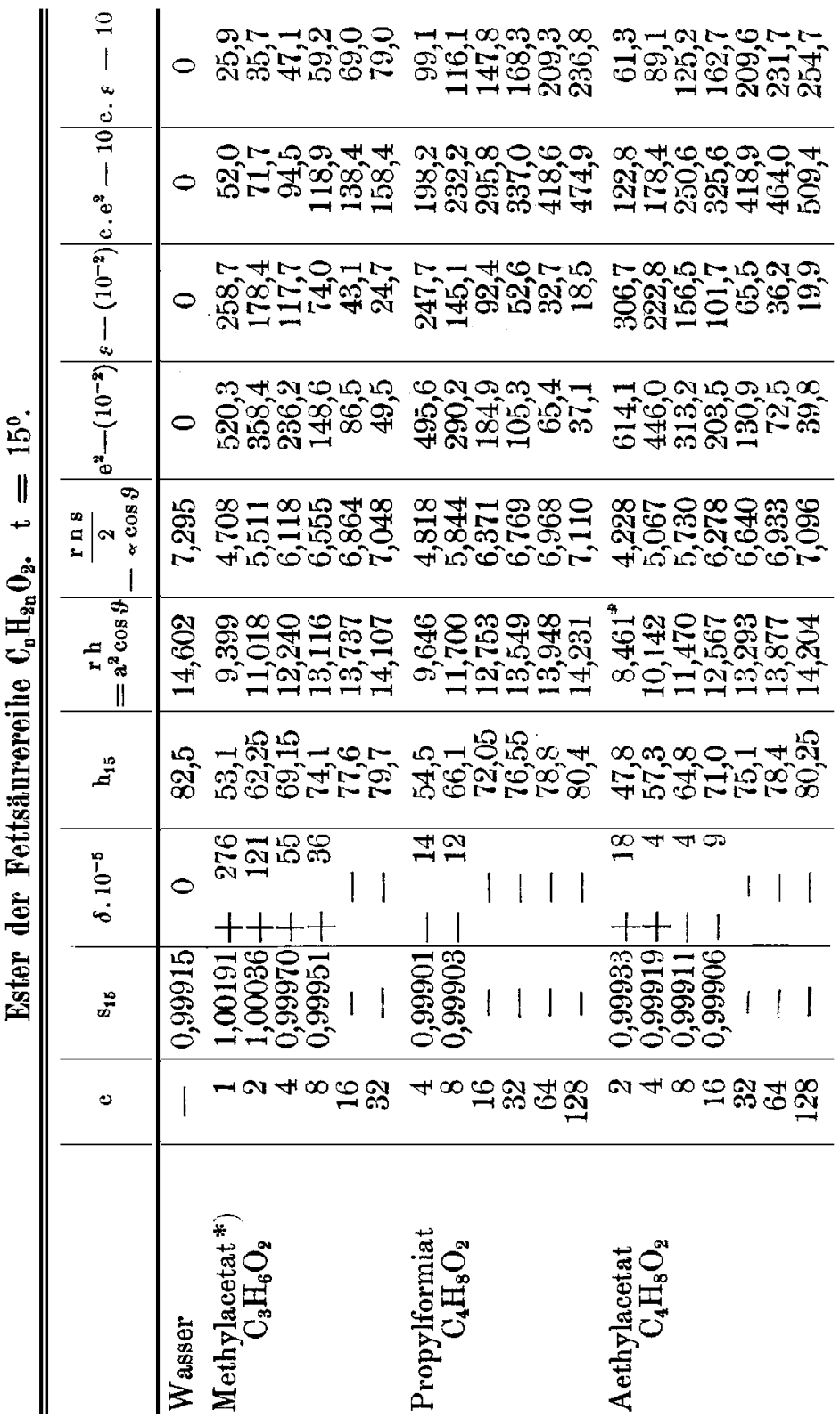




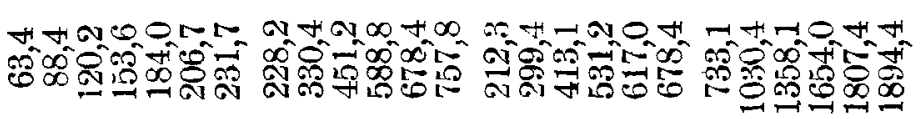

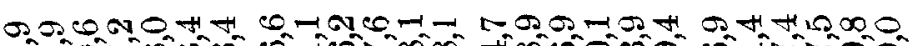
की

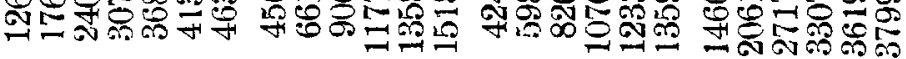

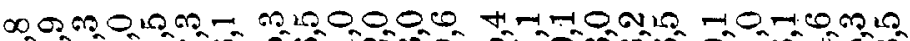

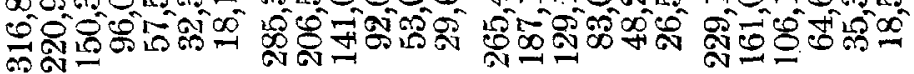

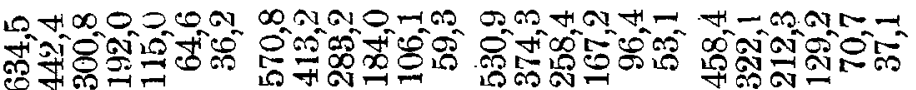

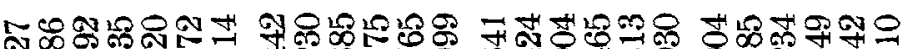

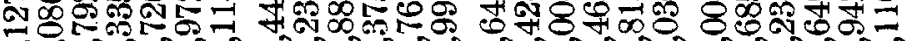

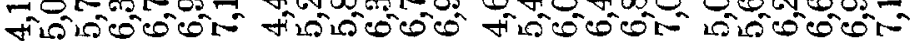

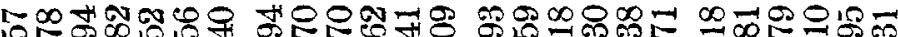

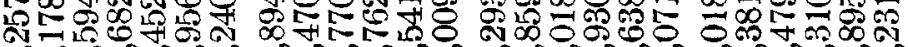

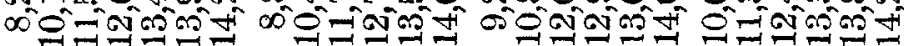

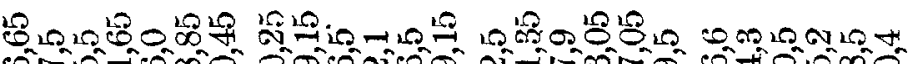
भi⿵

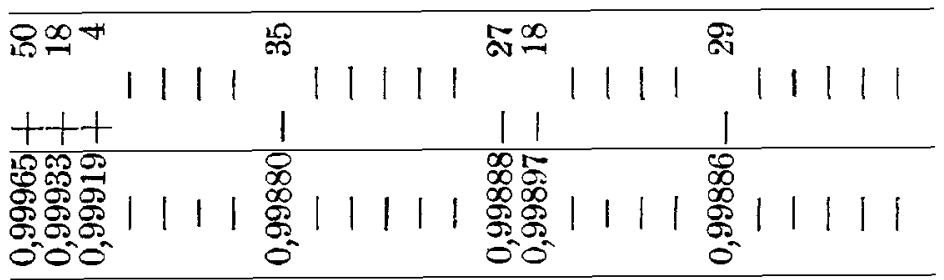

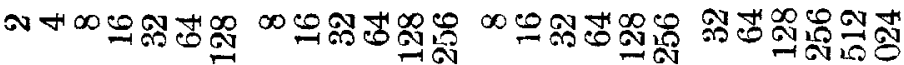
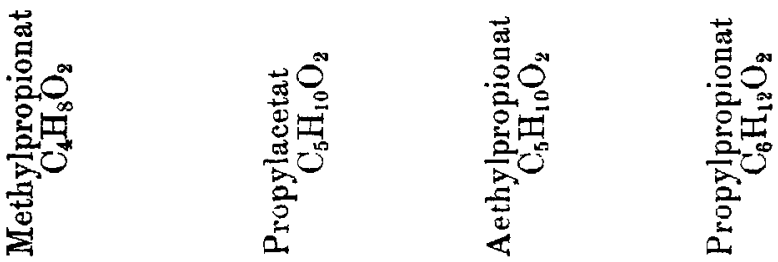


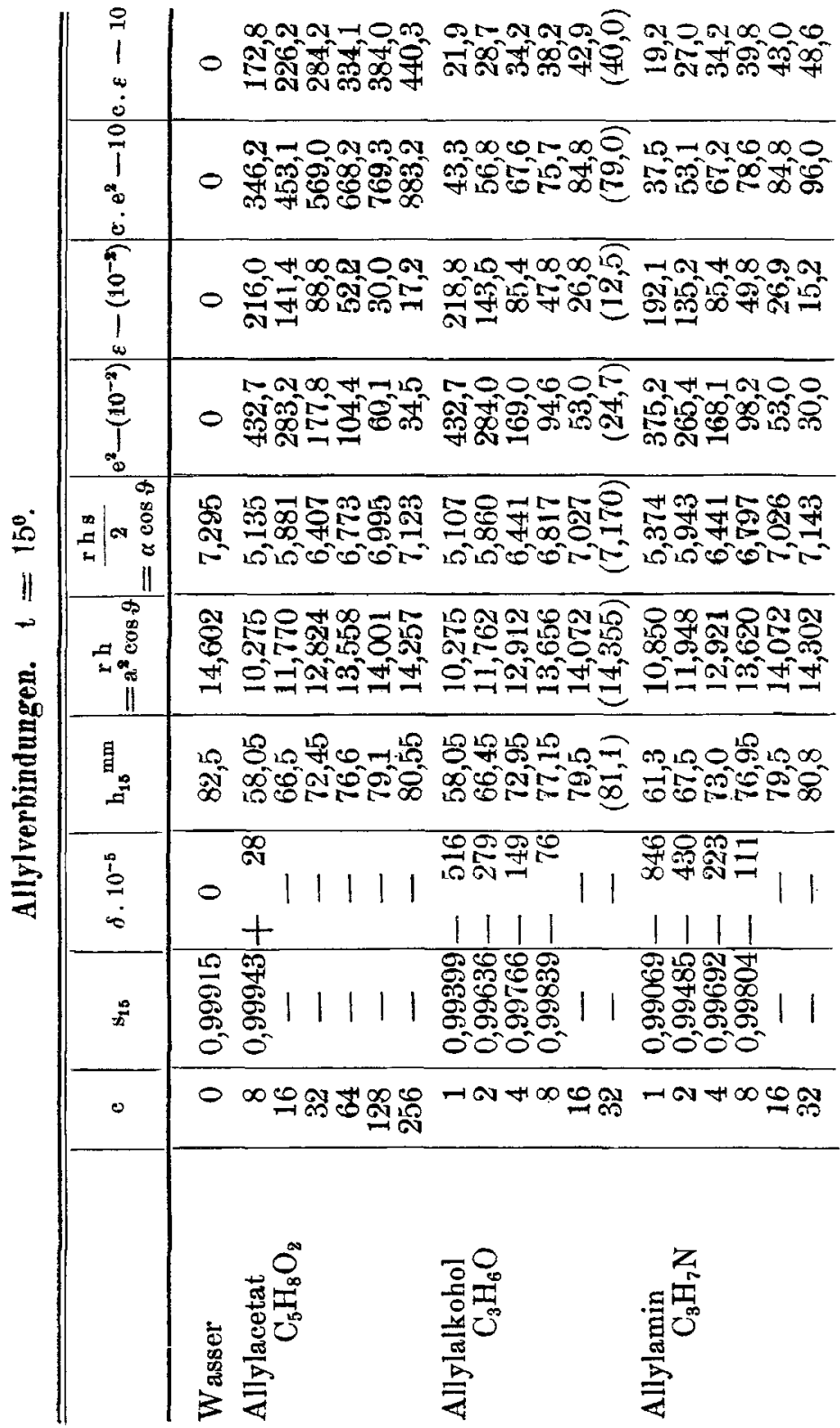




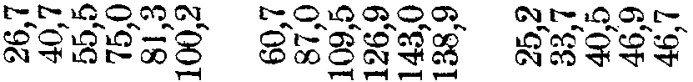

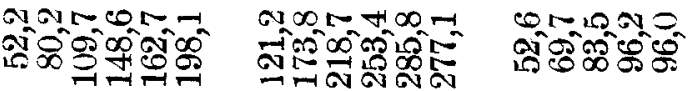

10100000000

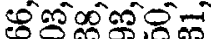

$\operatorname{cocos} \mathrm{cos}$

$\infty 010000$

बु

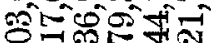

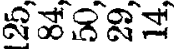

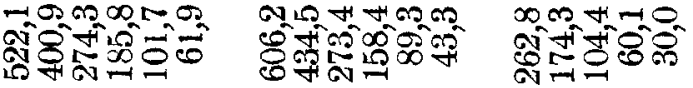

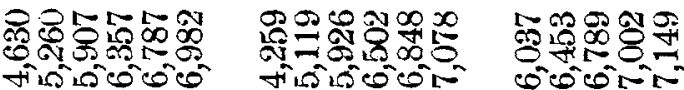

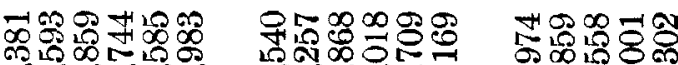

2n

$\sin$ -

$\infty 0=000$

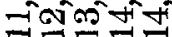

$0 . \infty 00100$

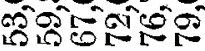

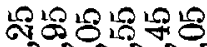
कीजिएक

$1000-\infty$

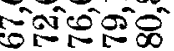

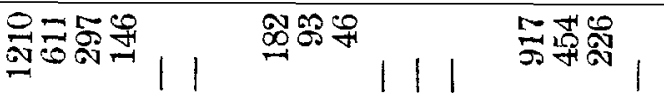

$+++$

\begin{tabular}{|c|c|c|}
\hline 1111 & 1 & $+1+$ \\
\hline 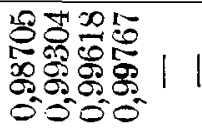 & 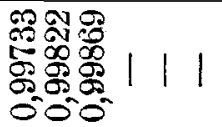 & 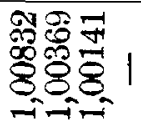 \\
\hline
\end{tabular}

-
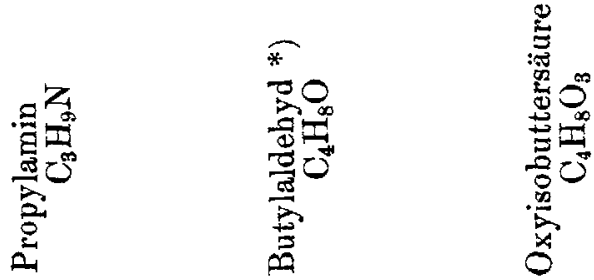


\begin{tabular}{|c|c|}
\hline 1 & 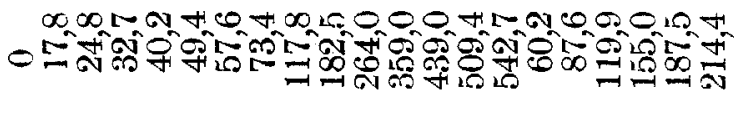 \\
\hline$\underset{\substack{0 \\
0 \\
0}}{1}$ & 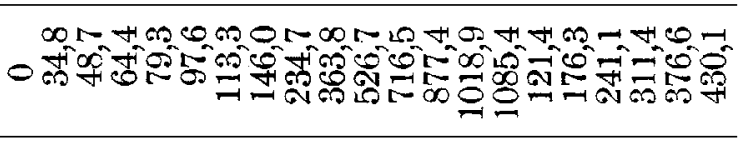 \\
\hline 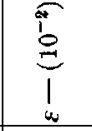 & 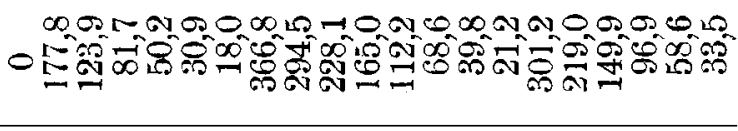 \\
\hline$\stackrel{9}{1}$ & 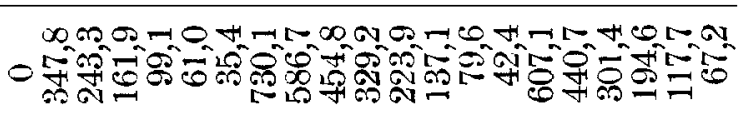 \\
\hline 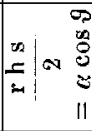 & 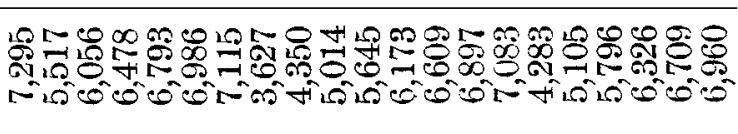 \\
\hline 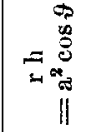 & 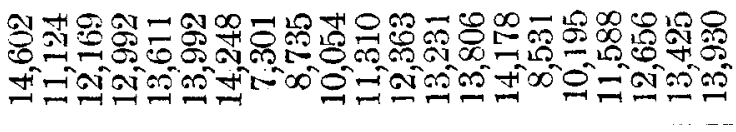 \\
\hline مِ & 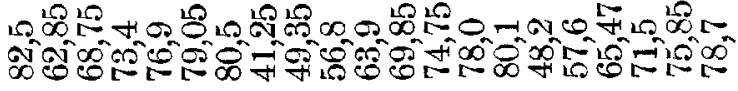 \\
\hline$\stackrel{i}{\stackrel{b}{i}}$ & 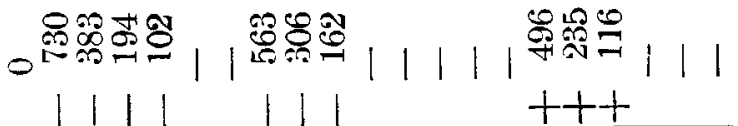 \\
\hline$\stackrel{0}{n_{n}^{2}}$ & 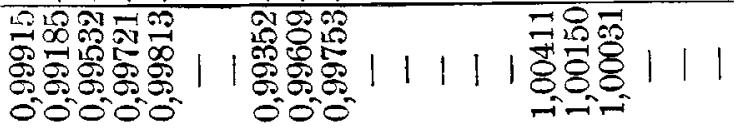 \\
\hline 0 & | - এ + \\
\hline & 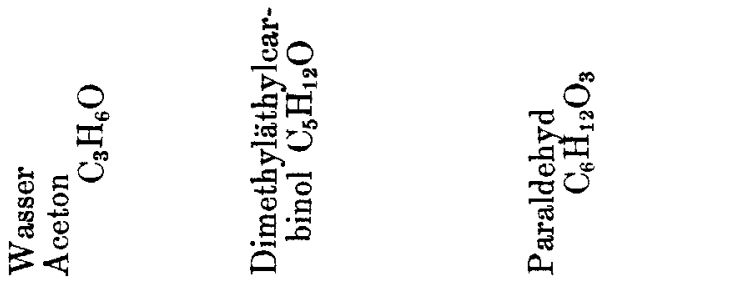 \\
\hline
\end{tabular}




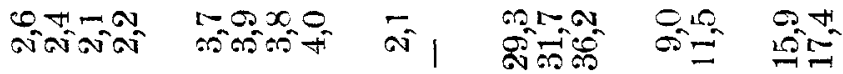

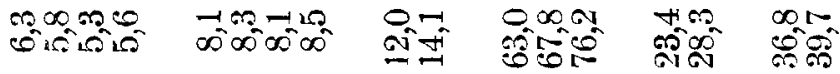

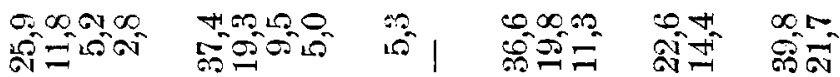

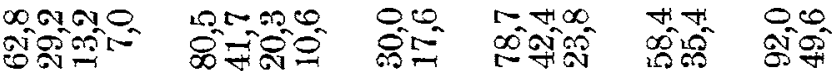

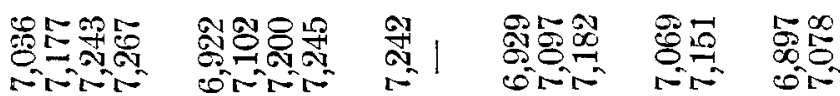

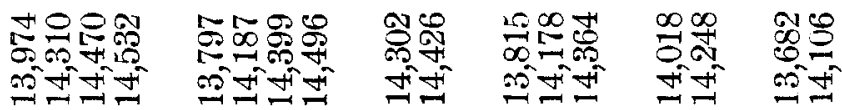

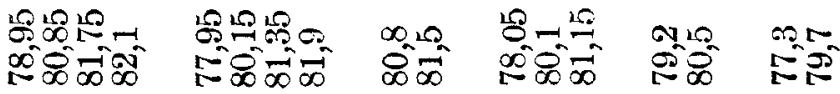

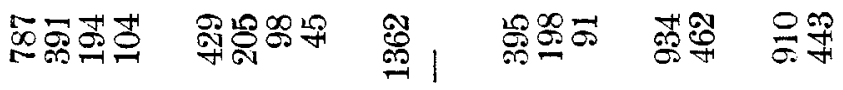

\begin{tabular}{|c|c|c|c|c|c|}
\hline$++1+$ & $+t+t$ & $t$ & $+1+$ & $+十$ & +1 \\
\hline 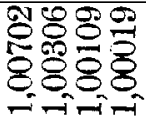 & 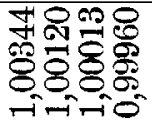 & $\frac{5}{0}$ & 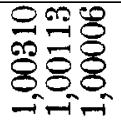 & 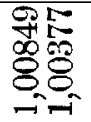 & 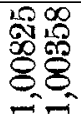 \\
\hline 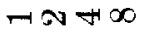 & $\pi \infty+\infty$ & $+\infty$ & $\infty 00$ & $+\infty$ & $\pi \infty$ \\
\hline
\end{tabular}
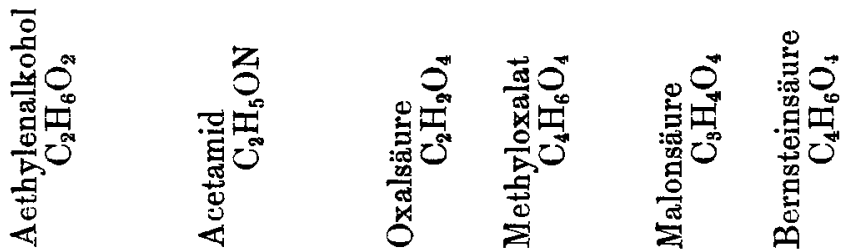


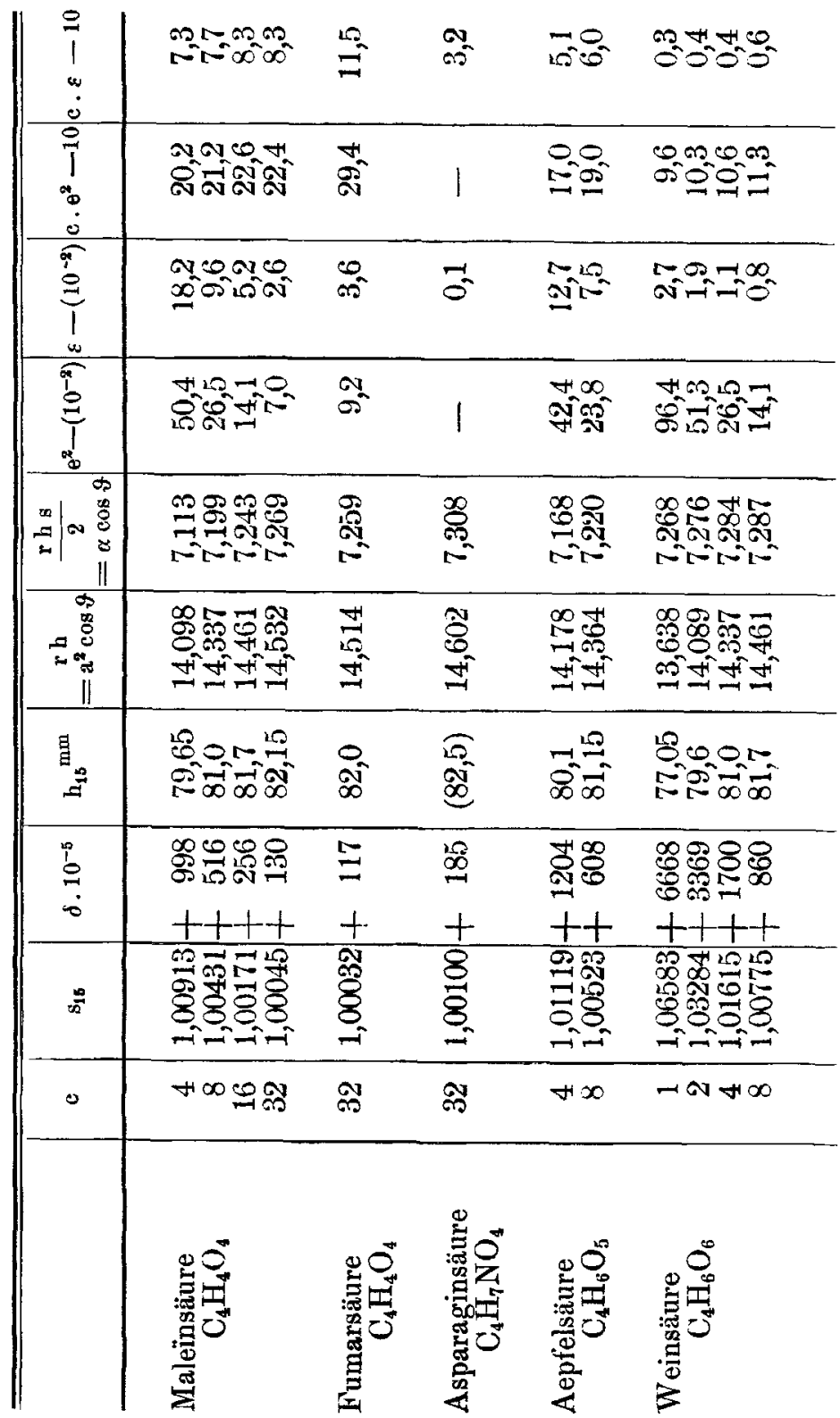


Die Betrachtungen, welche wir einleitend vorausschickten, sollen uns bei der Besprechung der Beobachtungsresultate leiten. Wir wollen hierbei als willkommene Ergänzung die Ergebnisse älterer Arbeiten ${ }^{*}$ ) von Valson, $Q$ u incke, Volckmann u. s. w. über Salzlösungen heranziehen.

Wenn wir vornehmlich die Werthe der Molekularcohäsion c $\varepsilon$ im Auge behalten, so ergiebt sich in der That, dafs diese Werthe mit zunehmender Verdünnung mehr und mehr zunehmen, um sich in hinreichenden Verdünnungen einem constanten Endwerthe zu nähern. Je nach der Natur des gelösten Stoffes tritt diese mehr oder weniger angenäherte Constanz in gröfseren oder geringeren Concentrationen ein. Für die meisten Salzlösungen wurde bereits, früher von Buliginski, Quincke und Volckmann festgestellt, dafs der Quotient aus den Werthen c $\varepsilon$ für Lösungen desselben Stoffes sich schon für die Concentrationen $c=1 \mathrm{zu} c=\infty$ sehr der Einheit nähert. Ein Blick auf die Tabellen, soweit dieselben sich auf die Körper der Oxalsäurereihe, Acetamid, Aethylenalkohol beziehen, zeigt, wie auch für diese Lösungen nahezu dasselbe gilt, wie für die Lösungen der Salze. Das B o y l e'sche Gesetz gilt angenähert hier bereits für höhere Concentrationen. Nehmen wir aber die Reihe der gewöhnlichen Alkohole, Fettsäuren, Ester, so ergiebt sich, dafs wir, namentlich je gröfser der Kohlenstoffgehalt, zu um so gröfseren Verdünnungen übergehen müssen, bis das Verhältnifs der Werthe $c \varepsilon$ sich der Einheit nähert. Die Verdünnung bei allen Stoffen so weit zu treiben, bis die Constanz der Werthe c $\varepsilon$ ersichtlich wird, haben wir unterlassen, weil namentlich beim Capillarimeter **) bei Ver-

*) Vgl. Valson, Compt. rend. 74, 103, 1872; Quincke, Pogg. Ann. 160, 337 und 560, 1877; Volckmanu, Wied. Ann. 1z, 353, 1882 und J. Traube, Journ f. pract. Chem. N. F. st, $206,1885$.

**) Das Stalagmometer wäre für die äufsersten Verdünnungen ge* eigneter gewesen. 
dünnungen, welche weniger als 1 bis $2 \mathrm{~mm}$ Steighöhenerniedrigung ergeben, gröfsere Beobachtungsfehler nur schwierig zu vermeiden sind. Die Werthe der Fettsäuren, Allylalkohol, Butylaldehyd, Oxyisobuttersäure *) u. s. w. deuten jedoch, wie mir scheint, auch bei diesen Stoffen genügend auf die Gültigkeit des Boyle'schen Gesetzes in hinreichend grofsen Verdünnungen hin.

Wir finden hier bei der Capillarität in dem Anwachsen der entsprechenden Constanten c $\varepsilon$ mit der Verdünnung gröfstentheils analoge Verhältnisse wieder wie beim electrolytischen Leitvermögen. Wenn auch von einer Gleichheit oder Proportionalität der entsprechenden Quotienten $c \varepsilon$ bezw. $\mu$ aus leicht erklärlichen Gründen nicht die Rede sein kann, so ergaben sich doch nicht nur in den Reihen der Salze, sondern auch der organischen Stoffe sehr nahe Beziehungen der beiden Eigenschaften.

O st w ald 's **) Arbeiten über das Leitvermögen wässeriger Lösungen der Fettsäuren, wie der Körper der Oxalsäurereihe und verwandter Säuren führten in Bezug auf den Einflufs der Concentration auf meist analoge Beziehungen wie in vorstehenden Tabellen, und eigene vorläufige Bestimmungen des Leitvermögens auch indifferenter Stoffe lassen keinen $Z$ weifel darüber aufkommen, dafs die Abnahme des Leitvermögens und der Molekularcohäsion mit der Concentration jedenfalls in derselben Ursache begründet ist.

Ja, die Beziehungen des Leitvermögens und der Capillarität erstrecken sich keineswegs nur auf den analogen Einflufs der Concentration. Mit demselben Recht oder Unrecht, mit welchem man elektrolytisch active und inactive Stoffe unter-

*) Interessant ist das Anwachsen der Molekularcohäsion mit wachsender Concentration bei Lösungen von Aethylenalkohol vgl. die analogen kryoscopischen Beobachtungen, Zeitschr. physik. Chem. 2, 498, 1888 und 4, 497, 1889.

**) Ostwald, Zeitschr. physik. Chem. 8, 170, 241, 369, 1889. 
scheidet, konnte Musculus *) bereits von capillar inactiven und activen Stoffen reden,

Je mehr ein Stoff capillar activ ist, d. h. je mehr er die Steighöhe des Wassers herabdrückt, um so weniger leitet im allgemeinen seine Lösung die Elektricität. Salze sind capillar inactiv, und electrolytisch activ; die meisten oder viele organische Stoffe capillar activ, electrolytisch inactiv. Je gröfser im allgemeinen die Capillarität der Lösung ist um so gröfser ist auch das Leitvermögen, wenn gleich die indifferenten organischen Stoffe vielfach eine Ausnahme von dieser Regel bilden. Der Eintritt der verschiedensten Gruppen, wie $\mathbf{C H}_{\mathbf{2}}$, $\mathrm{OH}, \mathrm{H}_{2}, \mathrm{Cl}, \mathrm{C}_{6} \mathrm{H}_{5}$ u. s. w. wirkt jedoch auch hier auf beide Constanten in gleichem Sinne. Dabei treten bei den Constanten der Capillarität oft kaum minder gewaltige Unterschiede auf, wie bei den Werthen des Leitvermögens. $1 / 100$ und $1 / 1000$ mancher organischen Stoffe üben auf die Steighöhe des Wassers einen gröfseren Einflufs aus, als ganze Procente der Salze ${ }^{*}$ ).

Bei dem electrolytischen Leitvermögen führen nun Arrhenius und Ostwald die Abnahme der Werthe $\mu$ mit zunehmender Concentration auf ganz bestimmte Dissociationsvorgänge zurück. Es ist hier nicht der Ort, auf die Dissociationshypothese näher einzugehen. Soviel aber steht fest, dafs dieselbe Annahme, welche dem Verhalten der Stoffe in Bezug auf die Elektricitätsleitung zu Grunde gelegt wird, bei der nahen Beziehung der Capillarität und des Leitvermögens auch für ihr Verhalten in capillarer Hinsicht in Betracht kommt.

*) Musculus, Chem. Centrbl. 1864 S. 922.

**) Hierin liegt, nebenbei bemerkt, die erhebliche Bedeutung der Capillaritätsconstanten für die analytische und speciell organische Chemie, die umsomehr hervorzuheben ist, als gerade diejenigen Stoffe, deren Lösungen ein vur schwaches elektrolytisches Leitvermögen besitzen, meist grof'se Empfindlichkeit im Capillarimeter zeigen, sodafs elektrolytisches Leitvermögen und Capillarität vor allem berufen $\mathbf{z u}$ sein scheinen, einander ergänzend, in der analytischen und organischen Chemie eine Rolle zu spielen. 
Nehmen wir eine Spaltung der Salze in Jonen an, so werden wir gezwungen, in den Lösungen der organischen Stoffe, schwimmende Radicale $\mathrm{CH}_{3}, \mathrm{C}_{2} \mathrm{H}_{5}, \mathrm{OH}, \mathrm{SH}, \mathrm{NH}_{2}, \mathrm{u}$. s. w. und Atome $\mathrm{H}, \mathbf{0}, \mathrm{S}, \mathrm{N}$ anzunehmen. Diese widersinnige Annahme wird gehoben durch die Associationshypothese*), d. h. diejenige Hypothese, welche die Abnahme des Leitvermögens und der Capillarität mit zunehmender Concentration auf eine Zunahme der Complexbildung zurückführt, ja die folgenden Betrachtungen werden uns zeigen, dafs uns die Capillaritätsconstanten sogar ein Mittel an die Hand geben, würden in vielen Fällen die Associationsvorgänge zahlenmälsig festzustellen.

Wenn wir nämlich die von der Dissociation unabhängigen constanten Endwerthe **) der Molekularcohäsion für verdünnteste Lösungen betrachten, so ergeben sich höchst auffallende Zahlenbeziehungen.

Die folgende Tabelle enthält die entsprechenden Werthe für die Fettsäuren, Alkohole und Säureester, unter $Q$ die Quotienten aus zwei benachbarten Werthen.

$\begin{array}{lrc} & \text { c } \varepsilon(-10) & \mathrm{Q} \\ \text { Ameisensäure } & 8,2 & 3,0 \\ \text { Essigsäure } & 24,8 & 3,0 \\ \text { Propionsäure } & 73,2 & 2,9 \\ \text { Buttersüure } & 215,0 & 1,0 \\ \text { Isobuttersäure } & 215,0 & \mathbf{2 , 8} \\ \text { Isovaleriansäure } & 605,4 & \\ \text { Methylalkohol } & 10,4 & \\ \text { Aethylalkohol } & 29,9 & 2,9 \\ \text { Propylalkohol } & 88,6 & 3,0 \\ \text { Isopropylalkohol } & 88,3 & 1,0 \\ \text { Isobutylalkohol } & 272,6 & 3,1 \\ \text { Isoamylalkohol } & \mathbf{7 3 7 , 6} & 2,7\end{array}$

*) Vgl. meine inzwischen erfolgte Veröffentlichnng, Ber. d. deutsch. chem. Ges. E8, 3582, 1890.

**) Als solche sind angenähert die Werthe zu betrachten, bei welchen die Steighöhenerniedrigung circa 3 bis $4 \mathrm{~mm}$ beträgt. Diese Werthe sind genauer, als diejenigen für gröfsere Verdünnungen. 


$\begin{array}{lcc} & \text { c } \varepsilon(-10) & \text { Q } \\ \text { Methylacetat } & 79,0 & \\ \text { Aethylacetat } & 254,7 & 3,2 \\ \text { Propylacetat } & 757,8 & 3,0 \\ \text { Methylpropionat } & 231,7 & 3,3 \\ \text { Aothylpropionat } & 678,4 & 2,9 \\ \text { Propylpropionat } & 1894,4 & 2,8\end{array}$

Aus den Tabellen ergiebt sich mit grofser Annäherung der Satz :

In homologen Reihen capillar activer Stoffe verhalten sich die constanten Endwerthe der Molekularcohäsionen fïr hinreichend verdünnte Lösungen wie $1: 3: 3^{2}: 3^{3} \ldots$ oder :

Der Druck, welchen die auf die Einheit der Fläche wirkenden gelösten Moleküle ausüben, wächst in homologen Reihen capillar activer Stoffe beim Eintritt einer $\mathrm{CH}_{2}$ gruppe im Verhältnifs $1: 3: 3^{2}: 3^{3} \ldots$

Dieser Satz ist gewifs höchst auffallend; ich will mich an dieser Stelle nicht in Vermuthungen über die Bedeuturig desselben ergehen. Ich zweifle nicht, dafs bei peinlichster Wiederholung der Versuche die nur wenig Abweichungen bei den letzten Gliedern der untersuchten 3 Reihen wegfallen werden; gerade hier können kleine Abweichungen in der Concentration, sowie geringe Unreinheiten der Substanz grofse Fehler veranlassen. Bei den bedeutenden Unterschieden der Werthe c. $\varepsilon$ kann an der sehr angenäherten Gültigkeit des Satzes kein $Z$ weifel sein.

Beim Leitungsvermögen hat sich ergeben, dafs gewisse Sätze $\left(\frac{\mu^{2}}{\mu_{1}{ }^{2}}=\frac{v}{v_{1}}\right)$ nur für die organischen Stoffe mit geringem Leitvermögen Geltung haben. Auch hier finden wir dasselbe. Obiger Satz gilt nur für die capillar activen Stoffe. Für die Oxalsäurereihe gilt er nicht, wenngleich auch hier - bei den 
Lösungen mit hohem spec. Gewicht vermuthlich einfache Zahlenbeziehungen nur deshalb nicht zum Vorschein kommen, weil hier secundäre Einflüsse, wie der Druck der durch das kleine Molekularvolumen der gelösten Stoffe in der Raumeinheit vorhandenen Ueberzahl von Wassertheilchen in Rechnung zu ziehen sind ${ }^{*}$ ).

Für die Associationstheorie sehr bedeutungsvoll scheinen mir die folgenden Erweiterungen des obigen Satzes zu sein : Wenn wir durch graphische Interpolation, wie dies zuerst für die Capillaritätsconstanten von $\mathrm{D}$ u c $\mathrm{I}$ a $\mathrm{u} \times \mathrm{x}$ geschehen ist, aus den Concentrationen $c$ und den Werthen $\varepsilon^{* *}$ ) die Concentrationen für gleichen capillaren Druck, also für \#isocapillare Lösungen berechnen, so erhalten wir Werthe, wie solche in den folgenden Tabellen veröffentlicht wurden. in bezeichnet die gelöste Molekülzahl für isocapillare Lösungen; $Q$ ist der Quotient aus den Werthen $m$.

*) Ich weifs, dafs ich mich mit den in dieser Abhandlung kurz and gedeuteten Ideeen über das Wesen der Capillarität bezw. Oberflächenspannung mit den herrschenden Theorien nicht iberall in Einklang befinde. Ich glaube aber, dals sich gerade hier mit kinetischen Vorstellungen viel erreichen lürst, und hoffe, in späteren Abhandlungen Gelegenheit zu haben, auf diesen Punkt zurückzukommen.

**) Ich hatte obenso die Constante $e^{2}$, oder c $\theta^{2}$ bezw. $c \varepsilon$ wählen können. Die Ergebnisse wären dieselben gewesen. 
응

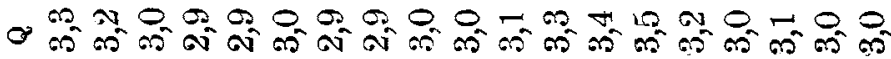

을

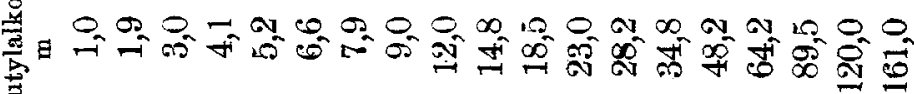

음

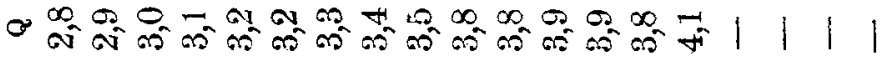

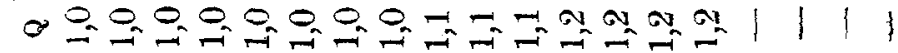

年

要

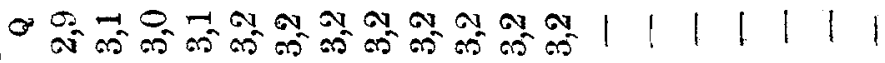

क

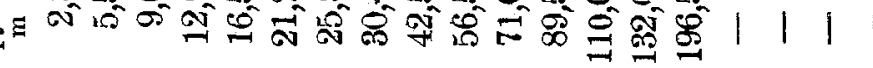

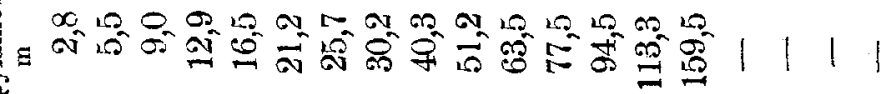

a d o 0 m n

घ $\infty$ कू

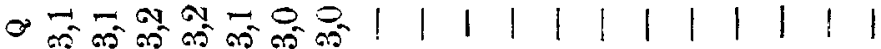

홍

$0,0, \infty, 0,0$

a

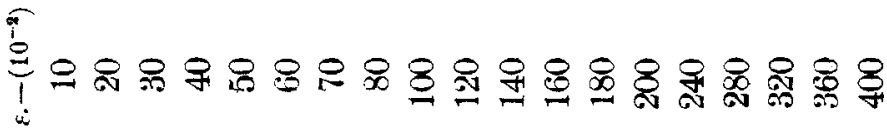



总

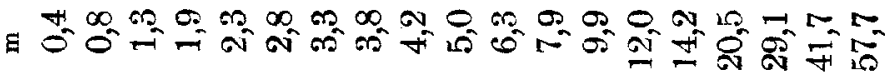

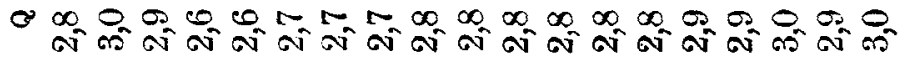
尝

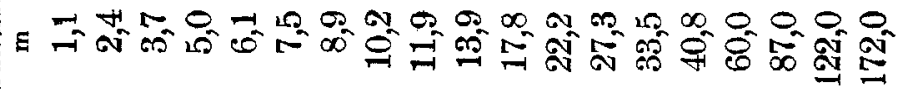

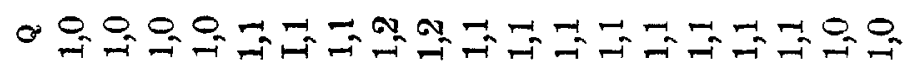

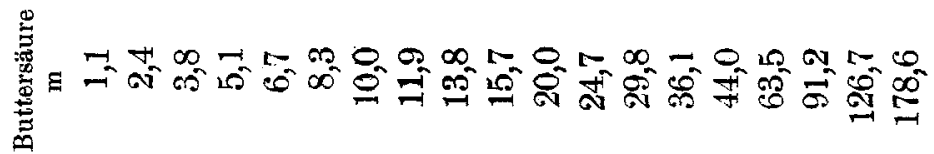

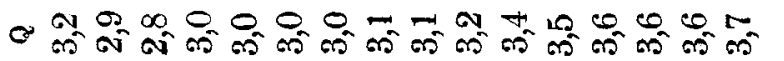
:

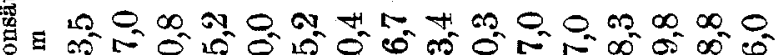

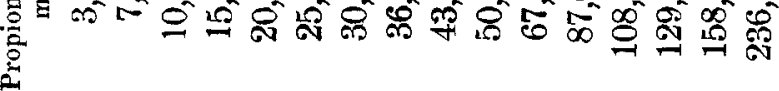

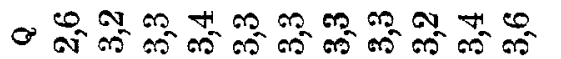

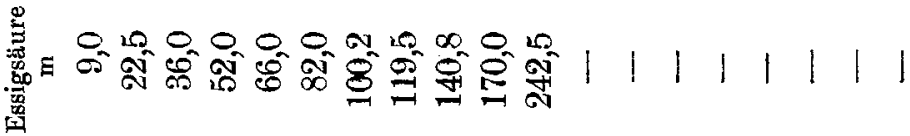
क लm 要

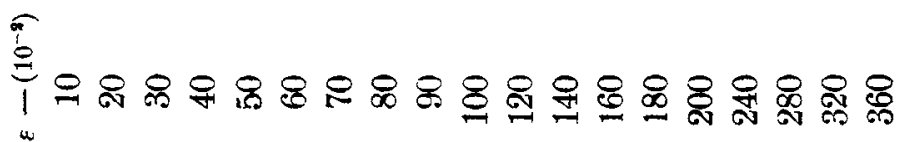


organischer Stoffe in wässerigen Lösungen.

范

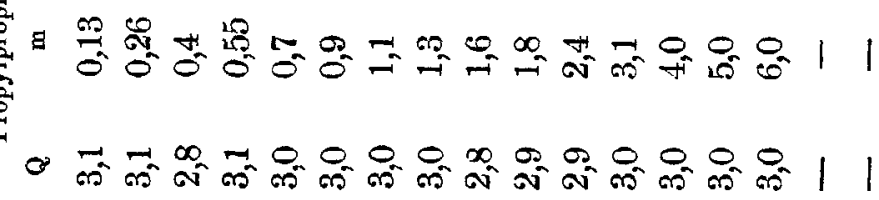

雚

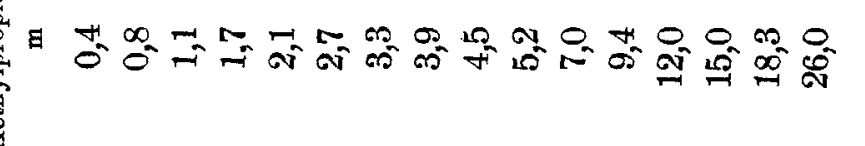

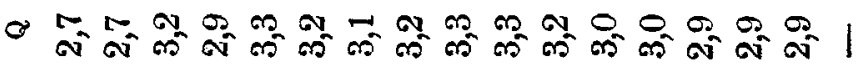

突

\#

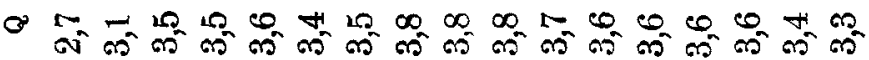

菜

a

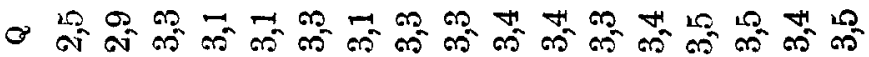

$\frac{+}{d}$

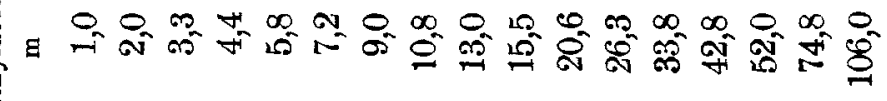

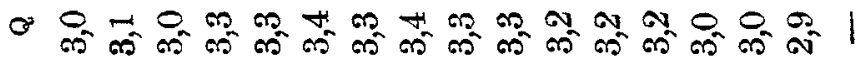

焉

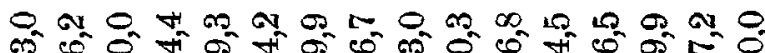

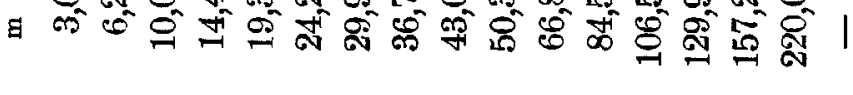

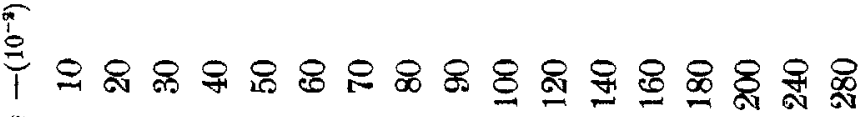

Annalen der Chemie 265. Bd. 
Von einigen Unregelmäfsigkeiten namentlich in den höheren Concentrationen einiger Stoffe absehend, ergeben sich die folgenden für die untersuchten Concentrationen gültigen Sätze :

1) In homologen Reihen capillar activer Stoffe ist das Verhältnifs der Molekülzahlen, welche gleichen Druck auf die Flächeneinheit ausüben, von der Anzahl der aufgelösten Molekïle nahezu unabhängig und constant. 2) In isocapitlaren Lösungen verhalten sich bei einem Unterschiede von n. $C H_{q}$ die relativen Molekülzahlen des gelösten Körpers wie $1: 3^{\text {n }}$.

Duclaux*) hat nach einer allerdings nicht einwandfreien Ausführung der Tropfmethode für Lösungen von gleichem Capillardruck die Capillaritätsconstanten aufgestellt. Er fand, dafs das Verhältnifs der Concentrationen bei gleichem Capillardruck mehr oder weniger angenähert constant war; er bezog jedoch diese Constanz fälschlich auf Gewichtsprocente, und unterliegt es wohl keinem Zweifel, dafs an Stelle der Constanz der relativen Gewichtsprocente die Quotienten aus dem gelösten Molekulargewicht zu setzen sind. Einfache Zahlenverhältnisse konnten sich in Folge der Fehler der Methode bei den Versuchen Ducla ux' nicht ergeben.

Nehmen wir an, dafs mit zunehmender Concentration eine Associirung der Moleküle zu Aggregaten stattfindet, so läfst der Umstand, dafs der Seite 45 ausgesprochene Satz auch für höhere Concentrationen Gültigkeit behält, kaum eine andere Deutung zu als die, welche in dem folgenden Satze iliren Ausdruck erhält.

Für solche (isocapillare) Lösungen verschiedener zunächst homologer Stoffe, welche auf die Einheit der Flüssigkeitsoberffäche einen gleich grofsen Druck ausüben, erfolgt die Association von Molekülen zu Molekiulaggregaten proportional den in der Lösung vorhandenen einfachen Molekülzahlen;

*) Ducla ux, Ann. Chim. Phys. [5] 8, 76. 1878. 
in isocapillaren Lösungen ist das Verhältnils der complexeren Moleküle gleich dem der einfachen Molekïle.

Diesem allerdings hyeothetischen, aber wie mir scheint sehr wahrscheinlichen Salze *) würde sich ein zweiter Satz an die Seite stellen.

Für die verdünntesten Lösungen von beisp. Isobutylalkohol oder Buttersäure gilt das B o yl e'sche Gesetz; d. h. eine Verdoppelung der Molekülzahl bewirkt eine Yerdoppelung der Steighöhenerniedrigung; in den höheren Concentrationen von $c=1$ bis 8 finden wir nun für Buttersäurelösungen die Steighöhen $37,2 \mathrm{~mm}, 46,05 \mathrm{~mm}, 55,22 \mathrm{~mm}$ und $63,42 \mathrm{~mm}$; für Lösungen von Isobutylalkohol $30,85 \mathrm{~mm}, 40,95 \mathrm{~mm}, 50,95 \mathrm{~mm}$ und $60,95 \mathrm{~mm}$; in der höheren Concentration bewirkt also eine Verdoppelung der Molekühlzahl keine Verdoppelung der Steighöhenerniedrigung, sondern einen gleich grofsen Zuwachs derselben. Die Curve wächst in grofsen Verdünnungen geometrisch, in grofsen Concentrationen arithmetisch. Diese Beobachtung, welche wohl für die capillaractiven Stoffe in entsprechend grofsen Concentrationen allgemein zutreffen dürfte, würde, dle Richtigkeit der Associationshypothese vorausgesetzt, zu dem Satze führen :

Mit zunehmender Concentration einer Lösung erfolgt die Association der Molekiule proportional der Zahl der gelösten Moleküle.

Die Capillarität würde demnach bier zu weit einfacheren Zahlenbeziehungen bezüglich der in Lösungen erfolgenden Dissociationsvorgänge, führen als das electrolytische Leitvermögen aus welchem man zuweitgehende Schlüsse über die gezogen hat.

Unser Zahlenmaterial erlaubt uns, die Beziehung der

*) Osmotischer Druck und Leitvermögen führen anscheinend zu demselben Salze. 
Capillarität zur Constitution aufser auf die $\mathbf{C H}_{2}$-Gruppe auch auf einige andere Gruppen auszudehnen.

Die Capillaritätsconstanten von Lösungen isomerer Fettsäureester und Fettsäuren sind angenähert gleich grofs.

Die folgende Tabelle zeigt das Verhältnifs der Molekülzahlen für gleiche Capillarität.

Propionsäure Buttersäure Methylpropionat Propylformiat

Methylacetat Aethylacetat Aethylacetat Methylpropionat

$\begin{array}{ccccc}\varepsilon-\left(10^{-2}\right) & Q & Q & Q & Q \\ 10 & 1,2 & 1,1 & 1,1 & 1,0 \\ 20 & 1,1 & 1,2 & 1,1 & 1,0 \\ 30 & 1,1 & 1,2 & 1,1 & 1,0 \\ 40 & 1,1 & 1,2 & 1,1 & 1,1 \\ 50 & 1,0 & 1,2 & 1,2 & 1,1 \\ 60 & 1,0 & 1,2 & 1,2 & 1,1 \\ 70 & 1,0 & 1,1 & 1,1 & 1,1 \\ 80 & 1,0 & 1,1 & 1,2 & 1,1 \\ 90 & 1,0 & 1,1 & 1,1 & 1,0 \\ 100 & 1,0 & 1,0 & 1,1 & 1,0 \\ 120 & 1,0 & 1,0 & 1,1 & 1,0 \\ 140 & 1,0 & 0,9 & 1,1 & 1,0 \\ 160 & 1,0 & 0,9 & 1,1 & 1,0 \\ 180 & 1,0 & 0,8 & 1,0 & 1,0 \\ 200 & 1,0 & 0,9 & 1,0 & 0,9 \\ 240 & 1,1 & 0,9 & 1,0 & 0,8\end{array}$

Es ist gewifs beachtenswerth, dafs isomere Ester und Säuren gleiche Capillarität zeigen, dennoch scheint die Verkeltung der Atome bei manchen Isomeren auch in verdünnten Lösungen eine Rolle zu spielen.

So zeigen zwar die beiden Propylalkohole und Buttersäuren, desgleichen in den verdünnten Lösungen Aceton und Allylalkohol sehr angenähert gleiche Capillarität; dagegen aber verlaufen die Curven bei Isoamylalkohol und Dimethyläthylcarbinol sehr verschieden, wenigstens ist für $c=256$ noch keine Uebereinstimmung erreicht.

Die folgende Tabelle zeigt für isocapillare Lösungen 
einiger Propyl- und Allylverbindungen die gelösten Molekülzahlen $\mathbf{m}$ und den Quotienten Q.

\begin{tabular}{|c|c|c|c|c|c|c|c|c|c|}
\hline & $\begin{array}{l}\text { Allyl- } \\
\text { acetat }\end{array}$ & & $\begin{array}{l}\text { Propyl- } \\
\text { acetat }\end{array}$ & $\begin{array}{l}\text { Allyl- } \\
\text { alkohol }\end{array}$ & & $\begin{array}{l}\text { Propyl- } \\
\text { alkohol }\end{array}$ & $\begin{array}{l}\text { Allyl- } \\
\text { amin }\end{array}$ & & $\begin{array}{l}\text { Propyl } \\
\text { amin }\end{array}$ \\
\hline$\varepsilon-\left(10^{-z}\right)$ & $\mathrm{m}$ & $Q$ & $\mathrm{~m}$ & $\mathrm{~m}$ & $\mathbf{Q}$ & m & $\mathbf{m}$ & $Q$ & $\mathrm{~m}$ \\
\hline 10 & 0,6 & 1,5 & 0,4 & 6,2 & 2,2 & 2,8 & 5,3 & 1,7 & 3,2 \\
\hline 20 & 1,2 & 1,7 & 0,7 & 12,0 & 2,2 & 5,5 & 11,3 & 2,2 & 5,0 \\
\hline 30 & 2,0 & 2,0 & 1,0 & 18,0 & 2,0 & 9,0 & 18,6 & 2,4 & 7,8 \\
\hline 40 & 2,9 & 2,1 & 1,4 & 25,8 & 2,0 & 12,9 & 25,5 & 2,2 & 11,3 \\
\hline 50 & 3,9 & 2,0 & 1,9 & 33,5 & 2,0 & 16,5 & 32,2 & 2,0 & 15,9 \\
\hline 60 & 4,7 & 2,1 & 2,5 & 41,9 & 2,0 & 21,2 & 41,0 & 2,1 & 19,4 \\
\hline 70 & 5,8 & 2,0 & 2,9 & 50,6 & 2,0 & 25,7 & 49,8 & 2,2 & 23,0 \\
\hline 80 & 7,0 & 2,1 & 3,3 & 59,6 & 2,0 & 30,2 & 59,1 & 2,2 & 26,5 \\
\hline 90 & 8,1 & 2,1 & 3,9 & 68,8 & - & - & 69,9 & 2,3 & 30,8 \\
\hline 100 & 9,5 & 2,1 & 4,5 & 78,4 & 1,9 & 40,3 & 81,0 & 2,2 & 36,0 \\
\hline 120 & 12,5 & 2,1 & 6,0 & 100,0 & 2,0 & 51,2 & 106,2 & 2,1 & 49,8 \\
\hline 140 & 15,9 & 2,0 & 7,9 & 124,0 & 2,0 & 63,5 & 137,0 & 2,1 & 65,0 \\
\hline 160 & 19,7 & 2,0 & 10,0 & 150,0 & 1,9 & 77,5 & 177,3 & 2,1 & 84,0 \\
\hline 180 & 24,0 & 1,9 & 12,3 & 181,0 & 1,9 & 94,5 & 223,0 & 2,1 & 103,8 \\
\hline 200 & 28,2 & 1,9 & 15,0 & 216,0 & 1,9 & 113,3 & - & - & - \\
\hline
\end{tabular}

Die Molekülzahlen der Propyl- und Allylverbindungen stehen in isocapillaren Lösungen im Verhältnils $1: 2$.

Von sonstigen Verbindungen, welche sich um 2 Wasserstoffatome unterscheiden, wurden untersucht Butylaldehyd Isobutylalkol, Propylalkohol - Aceton, Bernsteinsäure - Maleïnsäure und Aethylenalkohol - Essigsäure.

Bei den ersten 3 Paaren von Verbindungen zeigt sich in der That wenigstens für die verdünnten Lösungen sehr angenähert das obige Molekularverhältnifs 1 : 2 bei gleicher Capillarität. Dagegen hat Aethylenalkohol, obwohl wasserstoffreicher als Essigsäure, eine erheblich gröfsere Capillaritätsconstante. Allerdings dürfen Stoffe mit derart hohen Capillaritätsconstanten wie Aethylenalkohol in Bezug auf die hier gefundenen Regelmäfsigkeiten zunächst eben so wenig in $\mathrm{Be}-$ tracht gezogen werden, als für die electrolytisch gefundenen Gesetzmäfsigkeiten, um so weniger, als der Sauerstoff in $\mathrm{Hy}-$ 
droxylgruppen sich zweifellos anders verhält als einfach gebundener Sauerstoff.

Für das Sauerstoffatom konnten zunächst bei dem vorliegenden Material keine besonderen Regelmäfsigkeiten beobachtet werden, aufser dafs der Eintritt einer Hydroxylgruppe in allen beobachteten Fällen eine Erhöhung der Capillarität zur Folge hat.

Dieselbe ist aber verschieden sowohl von Bernsteinsäure Aepfelsãure und Aepfelsäure - Weinsäure, wie von Aethylalkohol - Aethylenalkohol und Buttersäure - Oxyisobuttersäure. Ebenso ergiebt der Vergleich von Butylaldehyd Buttersäure, Propionsäure - Aceton, Propylpropionat - Paraldehyd keine regelmäfsigen Beziehungen.

Bemerkenswerth ist der übereinstimmende Curvenverlauf für Allylamin - Allylalkohol, Propylamin - Propylalkohol, doch übt keineswegs die Amidogruppe allgemein den gleichen Einflufs wie die Hydroxylgruppe auf die Capillarität aus, wie das Verhalten des (allerdings capillar inactiven) Acetamids zu Essigsäure zeigt.

Mit dem Eintritt eines Kohlenstoffatoms finden wir bei den capillar activen Stoffen stets eine Erniedrigung der Capillarität verbunden. Für Buttersäure - Allylacetat, wie Aethylalkohol oder Aceton - Allylalkohol haben wir das ungefähre Verhältnifs $1: 1,6$ bis 1,7, für Propionsäure - Butylaldehyd $1: 2$, dagegen sind die Werthe $e^{2}$ für Propionsäure etwa 20 mal so gering wie die des Aethylenalkohols, und ebenso unregelmäfsig verhält sich Maleïnsäure - Malonsäure zu einander.

Alles in allem betrachtet, lassen sich zur Zeit noch wenig sichere Schlüsse über die Beziehungen der Capillarität zur Constitution der Verbindungen ziehen. Die vorhandenen Regelmärsigkeiten in den Reihen der capillar activen Stoffe deuten aber auf recht eigenthümliche Verhältnisse hin. Es dürfte sich lohnen, denselben weiter nachzuforschen, wobei namen- 
lich der gröfseren Einfachheit halber in capillarer Hinsicht hervorragend active Stoffe heranzuziehen wären. Anscheinend gehört die Capillarität, um mich eines Ausdrucks von Ost wald *) zu bedienen, zu den "colligativen * Eigenschaften, d. h. solchen Eigenschaften, bei denen der Zahlenwerth für gewisse Atomcomplexe gleich grofs ist. Diese Annahme würde auch am ehesten unseren Anschauungen über das Wesen der Capillarität entsprechen.

Mittheilung aus dem chemischen Institut der Universität Halle a./S.

\section{Zum Nachweis des Arsens;} von Joh. Thiele**).

1) Unterphosphorige Säure als Reagens auf Arsen.

Bereits früher ist beobachtet worden ***), dafs Arsen durch unterphosphorige Säure beim Einkochen metallisch niedergeschlagen werde. Wie ich gefunden habe, geht diese Reduction in salzsaurer Lösung auch ohne einzukochen und mit nicht sehr concentrirter unterphosphoriger Säure leicht von statten; sie giebt daher ein bequemes Mittel, Arsen auch in Gegenwart von Antimon und Zinn rasch nachzuweisen. Zur Ausführung der Reaction wird die zu prüfende Lösung, welche das Arsen als Arsensäure oder arsenige Säure enthalten kann, mit Salzsäure stark sauer gemacht und auf je

*) Ostwal d, Zeitschr. f. physik. Chem. 3, 414, 1889.

**) Aus des Verf. Inauguraldissertation. Halle a./S. 1890.

***) Gmelin-Kra ut's Handb. 2, 671. 